\title{
Tests of an Approximate Scaling Principle for Dynamics of Classical Fluids
}

\author{
Thomas Young ${ }^{\dagger}$ and Hans C. Andersen* \\ Department of Chemistry, Stanford University, Stanford, California 94305 \\ Received: October 4, 2004; In Final Form: November 11, 2004
}

\begin{abstract}
We used molecular dynamics computer simulations to test an approximate scaling principle that conjectures that two equilibrium atomic liquids have very similar dynamical properties if they have the same density and similar static pair correlation functions when the length scales of the two liquids are adjusted appropriately, even if they have different interatomic potentials and different temperatures. The simulations were performed on two types of model atomic liquids at various temperatures at the same density. In the first type, the interatomic potential is the Lennard-Jones potential (LJ). In the second type, the interatomic potential is the repulsive part of the Lennard-Jones potential (RLJ). We identified pairs of systems that have very similar pair correlation functions despite the fact that they had different potentials. Each pair consisted of an LJ liquid at a specific temperature and a corresponding RLJ liquid at a lower temperature. We compared various time correlation functions and transport coefficients of the two systems in each pair. Many dynamical properties are very similar in each pair, in accordance with the approximate scaling principle, whereas others are significantly different. The results indicate that certain dynamical properties are very insensitive to large changes in the interatomic potential that leave the pair correlation function largely unchanged, whereas other dynamical properties are much more sensitive to such changes in the potential. The transport coefficients for diffusion and viscosity are among the dynamical properties that are insensitive to such changes in the potential, and this may be part of the reason transport properties of many fluids have been calculated or rationalized in terms of a simple hard sphere model of liquids.
\end{abstract}

\section{Introduction}

Much research on the dynamics of liquids, especially molecular dynamics simulation studies, is concerned with understanding the relationship between the interatomic potential of a liquid and the time correlation functions and transport properties of the liquid. Accordingly, molecular dynamics computer simulations have been performed with a variety of different types of potentials. ${ }^{1-4}$ On the other hand, several formally exact kinetic theories ${ }^{5-11}$ have expressed the time correlation functions of a fluid in terms of the static correlation functions of the fluid with no direct reference to the interatomic potential. Such theories are described as "fully renormalized". The static correlation functions that appear in "fully renormalized" theories include the 2-point correlation function for density fluctuations, the 3-point function, the 4-point function, etc.

In the first few approximations that can be developed by using a formally exact theory (e.g., a mean field theory, a short time approximation, and a binary collision approximation, and even some versions of mode coupling theory), the only static correlation function that appears is the 2-point correlation function or pair correlation function. This led us to conjecture that different fluids might have very similar dynamical properties if they have the same density and similar static pair correlation functions, even if their potentials and temperatures were very different. In this paper we present a formulation of this conjecture, which we call an approximate scaling principle, based on an exact scaling principle, and we test the conjecture

\footnotetext{
* Address correspondence to this author. E-mail: hca@stanford.edu.

† Current address: Department of Chemistry, Columbia University, 3000 Broadway, MC 3167, New York, NY 10027. E-mail: tyoung@chem.columbia.edu.
}

through the use of molecular dynamics computer simulations. A preliminary account of this work has been published. ${ }^{12}$

The approximate scaling principle we use makes assertions about a variety of dynamical properties of a fluid, including time correlation functions for density fluctuations and current correlations, as well as transport coefficients. There is a long history of the use of analogous principles to relate transport coefficients of different fluids to one another, especially when one of the fluids is a hard sphere fluid. ${ }^{13-21}$

We performed simulations of two types of atomic liquid to test the approximate scaling principle. One type consists of atoms interacting via a Lennard-Jones potential and the other type consists of atoms interacting via the repulsive part of the Lennard-Jones potential. These two potentials are significantly different in that the former has attractive forces while the latter does not and the former has a much longer range than the latter. The simulated fluids were at one density with temperatures that varied by a factor of almost three. One of the calculations was for the Lennard-Jones fluid near its triple point.

From the simulations we were able to identify four pairs of systems with very similar structure but different potentials. We call these pairs of systems corresponding states. ${ }^{22}$ We present a detailed analysis of the dynamical correlations of density fluctuations of these corresponding states and find surprising similarities in their behavior.

Section 2 derives an exact scaling principle for the dynamics of classical systems. Its form motivates the statement of an approximate scaling principle for classical systems with different potentials but similar structures. Section 3 discusses the simulations we performed. In section 4 , pairs of corresponding states are identified. Section 5 contains a detailed comparison of the 
dynamics of corresponding states. Section 6 discusses the conclusions and implications of the results.

\section{Exact and Approximate Scaling Principles}

We consider an equilibrium classical atomic fluid that has $N$ interacting point particles, each of mass $m$, in volume $V$, at temperature $T$, to which classical mechanics and classical statistical mechanics apply.

The static correlation functions of interest are equilibrium ensemble averages of products of the density of particles, $\left\langle\rho\left(\mathbf{R}_{\mathbf{1}}\right) \ldots \rho\left(\mathbf{R}_{\boldsymbol{m}}\right)\right\rangle$. Here

$$
\rho(\mathbf{R}) \equiv \sum_{i=1}^{N} \delta\left(\mathbf{R}-\mathbf{r}_{i}\right)
$$

where $\mathbf{r}_{i}$ denotes the position of particle $i$ and angular brackets denote the canonical ensemble average.

The time correlation functions of interest are equilibrium ensemble averages of products of two or more time dependent densities in single particle phase space. The densities of interest are the total density

$$
f(\mathbf{R}, \mathbf{P}, t) \equiv \sum_{i=1}^{N} \delta\left(\mathbf{R}-\mathbf{r}_{i}(t)\right) \delta\left(\mathbf{P}-\mathbf{p}_{i}(t)\right)
$$

and the density of particle $i$

$$
f_{i}(\mathbf{R}, \mathbf{P}, t)=\delta\left(\mathbf{R}-\mathbf{r}_{i}(t)\right) \delta\left(\mathbf{P}-\mathbf{p}_{i}(t)\right)
$$

where $\mathbf{r}_{i}(t)$ and $\mathbf{p}_{i}(t)$ denote the position and momentum of particle $i$ at time $t$. Of particular interest is the 2-point correlation function

$$
\left\langle f\left(\mathbf{R}_{1}, \mathbf{P}_{1}, t\right) f\left(\mathbf{R}_{2}, \mathbf{P}_{2}, 0\right)\right\rangle
$$

which is related to the coherent neutron scattering properties and various current correlation functions, and

$$
N^{-1} \sum_{i=1}^{N}\left\langle f_{i}\left(\mathbf{R}_{1}, \mathbf{P}_{1}, t\right) f_{i}\left(\mathbf{R}_{2}, \mathbf{P}_{2}, 0\right)\right\rangle
$$

which is related to incoherent scattering properties and the velocity autocorrelation function.

To formulate the scaling principles, we define a scaled length, scaled momentum, and scaled time.

To define a scaled length, we choose a system dependent constant $l$ with the dimensions of length, and let

$$
\tilde{\mathbf{r}}_{i}=l^{-1} \mathbf{r}_{i}
$$

with a similar scaling for $\mathbf{R}$. The length $l$ is chosen to be of the form

$$
l=a \rho^{-1 / 3}
$$

where $\rho=N / V$ is a system dependent quantity and $a$ has the same value for all systems being considered. Its choice is discussed below. We define scaled momenta as

$$
\tilde{\mathbf{p}}_{i}=\left(m k_{\mathrm{B}} T\right)^{-1 / 2} \mathbf{p}_{i}
$$

We define scaled time as

$$
\tilde{t}=l^{-1}\left(k_{\mathrm{B}} T / m\right)^{1 / 2} t
$$

The scaled lengths, momenta, and times are dimensionless. In effect we have chosen a system dependent set of units, and quantities denoted with a tilde are the dimensionless numerical values of quantities of interest when they are expressed in terms of those units. (Any quantity denoted with a tilde is to be understood as a value expressed in terms of these units.)

We note that the distribution function for scaled momenta in the canonical ensemble is independent of temperature. Therefore, the canonical ensemble joint distribution functions of the $N$ scaled momenta are the same for any two systems of $N$ particles.

2.1. Exact Scaling Principle. The exact scaling principle of interest is the following: Consider two atomic fluids, denoted $\mathrm{A}$ and $\mathrm{B}$, with the same $N$, each in a certain intensive thermodynamic state characterized by its values of $T$ and $\rho$. The values of $T, \rho$, and $m$, as well as the form of the interatomic potential energy function, are in general different in the two fluids. Suppose a common value of $a$ is chosen for scaling the length. (The specific value of $a$ will turn out not to be important.) The lengths and momenta are scaled as described above. If all the static correlation functions of the density are the same for two systems when expressed in terms of scaled length, i.e., if

$$
\tilde{\rho}_{n}{ }^{[\mathrm{A}]}\left(\tilde{\mathbf{R}}_{1}, \tilde{\mathbf{R}}_{2}, \ldots, \tilde{\mathbf{R}}_{n}\right)=\tilde{\rho}_{n}{ }^{[\mathrm{B}]}\left(\tilde{\mathbf{R}}_{1}, \tilde{\mathbf{R}}_{2}, \ldots, \tilde{\mathbf{R}}_{n}\right)
$$

for all values of $n(\geq 1), \tilde{\mathbf{R}}_{1}, \ldots, \tilde{\mathbf{R}}_{n}$, then the two systems have identical time correlation functions provided the latter are expressed in terms of the scaled length, scaled momenta, and scaled time.

The proof of the exact scaling principle is straightforward, and the details will be omitted. The essential ideas of the proof are the following. The scaling factors are in general different for different systems. The scaling of length ensures that the two systems have the same value of the scaled density and the scaled volume. The scaling of momentum ensures that they have the same equilibrium joint distribution function of scaled momenta. The assumption in eq 2 is strong enough to imply that the two systems have the same joint distribution function of the scaled particle coordinates. This implies that, when expressed in terms of scaled coordinates and momenta, the two systems' Hamiltonians divided by their respective values of $k_{\mathrm{B}} T$ differ by a constant. This implies a simple relationship between the Hamiltonian equations of motion for the scaled coordinates and momenta of the two systems. That relationship leads to the conclusion that when time is scaled in the way discussed above, the equations of motion of the two systems are identical in form. The conclusion of the theorem follows.

2.2. Approximate Scaling Principle. The approximate scaling principle can be formulated in the following way: Consider two atomic fluids with the same $N$, each in a certain intensive thermodynamic state characterized by its values of $T$ and $\rho$. The values of $T, \rho$, and $m$, as well as the form of the interatomic potential energy function, may be different in the two fluids. Suppose that for each system the lengths, momenta, and time are scaled as described above. If the scaled densities of the two systems are equal and the static pair correlation functions of the scaled density are very similar for the two systems when expressed in terms of scaled length, then their time correlation functions, when expressed in terms of scaled variables, are very similar even if their interatomic potentials are very different. (A pair of systems that have very similar static pair correlation functions when expressed in terms of scaled length will be called a pair of corresponding states.)

In order for this conjecture to be true, presumably both of the following statements would have to be true: (1) The dynamics are not very sensitive to the static correlation 
functions. (If small changes in the structure lead to only small changes in the dynamics, then a large change in the potential that leads to only a small change in the structure will lead to only a small change in the dynamics). (2) Either the static pair correlation function is the dominant correlation function for determining dynamics, or the fact that the static pair correlation functions of the two systems are similar also implies that the higher correlation functions are similar.

The approximate scaling principle is merely a conjecture, but one that can clearly be tested. The rest of this work contains a detailed analysis of our tests of this conjecture.

\section{Molecular dynamics simulations}

Molecular dynamics simulations were performed on systems of 500 particles at one density for a variety of temperatures and two different interatomic potentials. The atomic mass $m$ of the particles in the two systems was chosen to be the same.

3.1. Interatomic Potentials. The two potentials are closely related to the Lennard-Jones potential,

$$
u(r)=4 \epsilon\left[\left(\frac{\sigma}{r}\right)^{12}-\left(\frac{\sigma}{r}\right)^{6}\right]
$$

where $\epsilon$ has dimensions of energy and $\sigma$ has dimensions of length.

The first potential, which we will refer to as the LJ potential, is the Lennard-Jones potential truncated at $2.5 \sigma$ and shifted upward to make it continuous at the cutoff.

$$
\begin{gathered}
u_{\mathrm{LJ}}(r)=u(r)-u(2.5 \sigma) \quad \text { for } r \leq 2.5 \sigma \\
u_{\mathrm{LJ}}(r)=0 \quad \text { for } r \geq 2.5 \sigma
\end{gathered}
$$

The second potential is the repulsive part of the LennardJones potential. ${ }^{23}$ This potential is the Lennard-Jones potential truncated at $2^{1 / 6} \sigma$ and shifted upward to make it continuous at the cutoff. We will refer to it as the $R L J$ potential.

$$
\begin{gathered}
u_{\mathrm{RLJ}}(r)=u(r)-u\left(2^{1 / 6} \sigma\right) \quad \text { for } r \leq 2^{1 / 6} \sigma \\
u_{\mathrm{RLJ}}(r)=0 \quad \text { for } r \geq 2^{1 / 6} \sigma
\end{gathered}
$$

Unlike the LJ potential, this potential is nonnegative and has no attractive forces. It is also of much shorter range than the $\mathrm{LJ}$ potential.

We use $m, \epsilon$, and $\sigma$ as the fundamental units for mass, energy, and length and a unit for temperature such that Boltzmann's constant, $k_{\mathrm{B}}$, is dimensionless and equal to unity. ${ }^{3}$ The derived unit for time is $\tau \equiv\left(m \sigma^{2} / \epsilon\right)^{1 / 2}$. For a quantity with dimensions, the numerical value of the quantity when expressed in terms of this set of units will be called the "reduced" quantity and will be denoted by an asterisk; e.g., the reduced time $t^{*}$ corresponding to a time $t$ is $t^{*} \equiv t / \tau$, and the reduced temperature is $T^{*} \equiv$ $k_{\mathrm{B}} T / \epsilon$.

3.2. Scaling. In testing the approximate scaling principle, we will always be comparing two systems with the same density. Hence the scaling length $l$ in eq 1 is the same for the two systems. It is convenient to choose $a$ such that $l=\sigma$. Thus the scaled reduced length and the reduced length are equivalent. The scaled reduced time associated with the reduced time $t^{*}$ is simply given by $\tilde{t}^{*} \equiv\left(T^{*}\right)^{1 / 2} t^{*}$. Other quantities that have units containing powers of time are scaled in a consistent way; e.g., the diffusion constant $D$ has units of (length) ${ }^{2} /$ time. The reduced diffusion constant $D^{*}$ is defined so that $D=D^{*} \sigma^{2} / \tau$. Since $D$ has one inverse power of time, the scaled reduced diffusion coefficient is $\tilde{D}^{*}=D^{*} /\left(T^{*}\right)^{1 / 2}$. Thus we have $D=\tilde{D}^{*} \sigma^{2} / \tau_{\mathrm{s}}$ where $\tau_{\mathrm{s}}=\tau /\left(T^{*}\right)^{1 / 2}$ is the unit of scaled time, i.e., the value of the time when the scaled reduced time is unity.

3.3. Simulation Details. The molecular dynamics calculations used the velocity version of the Verlet algorithm ${ }^{24}$ with a time step of $0.008 \tau$. The simulated system consisted of 500 particles in a cubic volume with periodic boundary conditions. The reduced density $\rho^{*}=N \sigma^{3} / V$ for all systems studied was 0.85 .

We define a run as one constant energy molecular dynamics trajectory of a system with a given potential and given $N$ and $V$, starting from a configuration that has been equilibrated to a certain temperature $T$. The starting state for each run was constructed in the following way: (1) The particle positions were chosen in a manner described below. (2) The particle momenta were independently chosen at random from the Maxwell-Boltzmann distribution appropriate for the temperature. (3) The center of mass velocity was set to zero by subtracting the mean velocity of the particles from the velocity of each particle. (This eliminates the steady drift of the system, relative to the coordinate axes, that can introduce unphysical artifacts in calculated dynamic correlation functions.) The first run for a given potential and temperature used initial particle positions that were obtained by thorough equilibration of a simulated system for a long period of time. For each subsequent run for the same potential at the same temperature, the initial particle positions were chosen as the final positions of the previous run.

For each of the states studied, we performed 100 runs. The duration of each run was $800 \tau$. Static and dynamic correlation functions were calculated separately for each run. Then the data for all runs for the same potential and the same $T$ were averaged, and the statistical error of the average was estimated in the standard way by using the assumptions that the results of different runs were statistically independent. This is a good assumption given the length of the runs relative to the correlation times for the states concerned.

3.4. Correlation Functions. Several correlation functions were calculated. We use the terminology and the definitions of Boon and Yip $^{1}$ in most cases. The static correlation functions are the pair correlation function $g(r)$ and the structure factor $S(k)$. The dynamic correlation functions are the coherent intermediate scattering function $F(k, t)$, the incoherent intermediate scattering function $F_{\mathrm{s}}(k, t)$, the normalized velocity autocorrelation function $\psi(t)$, the longitudinal current correlation function $J_{1}(k, t)$, and the transverse current correlation function $J_{t}(k, t)$. We also calculated the incoherent or self-part of the longitudinal current correlation functions, which we denote $J_{\text {ls }}(k, t)$.

The functions $F(k, t), F_{\mathrm{s}}(k, t)$, and $\psi(t)$ are dimensionless, and so their values are unaffected by scaling. $F_{\mathrm{s}}(k, t)$ and $\psi(t)$ are equal to unity at $t=0$ as a result of their definitions. In the graphs below, the current correlation functions have been normalized to have the value of unity at $t=0$. These normalized functions are in fact equivalent to the corresponding scaled functions. One of the figures of $F(k, t)$ is normalized to unity at $t=0$ but merely for convenience.

Most of the time dependent correlation functions of interest are functions of the length of a wave vector $\mathbf{k}$. Data were collected for a specific set of $\mathbf{k}$ vectors whose lengths ranged from $0.75 \sigma^{-1}$ (the smallest value for which data could be collected, given the size of the system) to $7.50 \sigma^{-1}$. The wave vector $k=6.75 \sigma^{-1}$ is the one for which data were collected that is closest to the major peak of the static structure factor. 
TABLE 1: The Four Pairs of Corresponding States (CS) Investigated in This Work ${ }^{a}$

\begin{tabular}{ccl}
\hline CS & $T^{*}$ RLJ & $T^{*} \mathrm{LJ}$ \\
\hline 1 & 0.446 & 0.723 \\
2 & 0.723 & 1.0 \\
3 & 1.0 & 1.277 \\
4 & 1.277 & 1.554
\end{tabular}

${ }^{a}$ The pairs are labeled $1-4$. In each pair, $T^{*} \mathrm{RLJ}$ is the temperature of the system with the RLJ potential and $T^{*} \mathrm{LJ}$ is the temperature of the system with the LJ potential. All eight states had the same reduced density $\rho^{*}$ of 0.85 .

\section{Corresponding States}

4.1. Identifying Corresponding States. It is well-known, from work on the perturbation theory of liquid structure, ${ }^{23}$ that the LJ and RLJ liquids at the same density and temperature have similar static structures provided the density is high. In particular, the static structure factor as a function of wave vector $k$ has a similar shape for two such systems, the major difference being a slightly higher peak for LJ as opposed to the RLJ system. It is as if "turning on" attractions in the RLJ system, thereby converting it to an LJ system, changes the structure factor primarily in the height of the first peak and leaves other features largely unchanged. A similar change in an RLJ structure factor can also be accomplished merely by lowering the temperature, keeping the potential unchanged.

Thus, while an LJ and an RLJ fluid at the same temperature and same (high) density have similar structure factors, a closer similarity between two structure factors is obtained if one compares an LJ fluid with an RLJ fluid at a somewhat lower temperature. For example, an $\mathrm{LJ}$ fluid at $T=1.0$ has a very similar structure factor to an RLJ fluid at $T=0.723$.

By closely matching the first peaks of the static structure factor for RLJ systems at one reduced temperature and LJ systems at higher reduced temperatures, we were able to find four pairs of systems with very similar overall static structure factors. These pairs of systems are the pairs of corresponding states that were studied in this work. Their temperatures are given in Table 1. The four pairs will be referred to as CS1, CS2, CS3, and CS4.

4.2. Comparison of the Structures of Corresponding States. The static structure factors for the third pair of corresponding states, CS3, are compared in Figure 1. (The corresponding results for $\mathrm{CS} 2$ are in Figure 1 of a previous paper. ${ }^{12}$ ) The differences are barely noticeable in such a plot. Similar agreement is found for each of the other three pairs of corresponding states.

For all four pairs of corresponding states for values of $k \geq$ $3.75 \sigma$, the agreement of the static structure factors is very good. For the pair of corresponding states with the lowest temperature (CS1), the difference in amplitude of the first peak is not statistically significant. For the other pairs of corresponding states the magnitudes of the first peak are within $1 \%$ of each other. All pairs of data points in all four pairs of corresponding states for each $k \geq 3.75 \sigma$ are within $3 \%$ of each other. For all four pairs of corresponding states for values of $k<3.75 \sigma$, the absolute differences in the values of $S(k)$ are small. However, since the $S(k)$ values are small, the percentage differences are more substantial.

For future reference, a comparison of the structure factors for the corresponding states in the limit $\mathbf{k} \rightarrow 0$ should be discussed. The static structure factor data for the five smallest $\mathbf{k}$ vectors were fit to a quartic function of $|\mathbf{k}|$ to extract estimates

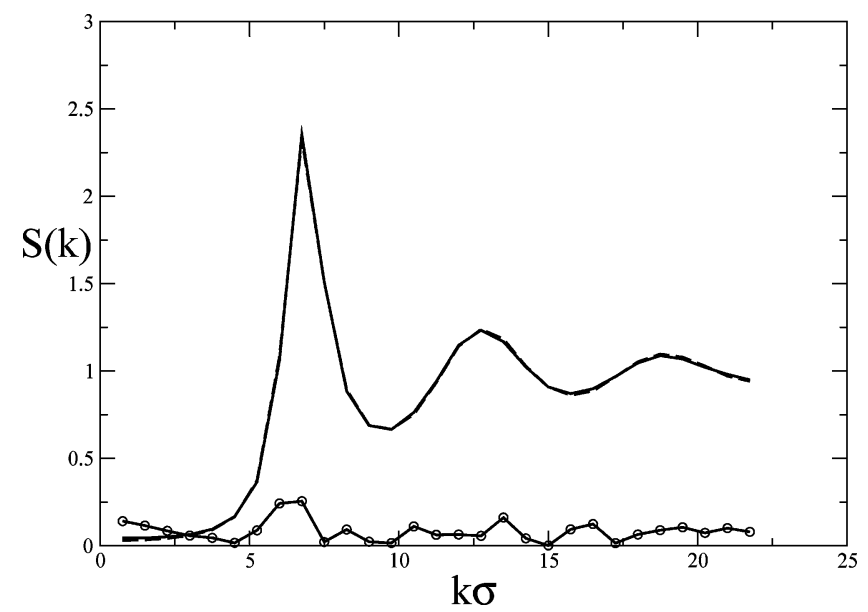

Figure 1. The static structure factor $S(k)$ as a function of $k \sigma$ for CS3. The RLJ system at $T^{*}=1.0$ (dashed line) and the LJ system at $T^{*}=$ 1.277 (solid line). These two curves are barely distinguishable in this figure. The lower curve with circular data points is a plot of 10 times the absolute value of the difference between the two upper curves.

TABLE 2: Properties Related to Sound Propagation in the Eight Systems Studied ${ }^{a}$

\begin{tabular}{lcccccc}
\hline \multicolumn{1}{c}{$T^{*}$} & potential & $\lim _{k \rightarrow 0} S(k)$ & $\rho^{*} \chi_{\mathrm{T}}^{*}$ & $c_{\mathrm{T}}^{*}$ & $\tilde{c}_{\mathrm{T}}^{*}$ & $\mathrm{CS}$ \\
\hline 0.446 & RLJ & 0.0204 & 0.0457 & 4.68 & 7.01 & 1 \\
0.723 & LJ & 0.0389 & 0.0538 & 4.31 & 5.07 & 1 \\
0.723 & RLJ & 0.0255 & 0.0353 & 5.32 & 6.26 & 2 \\
1.0 & LH & 0.0417 & 0.0417 & 4.90 & 4.90 & 2 \\
1.0 & RLJ & 0.295 & 0.0295 & 5.82 & 5.82 & 3 \\
1.277 & LJ & 0.0444 & 0.0348 & 5.36 & 4.74 & 3 \\
1.277 & RLJ & 0.0329 & 0.0258 & 6.23 & 5.51 & 4 \\
1.554 & LJ & 0.0470 & 0.0302 & 5.75 & 4.61 & 4
\end{tabular}

${ }^{a} T^{*}$ is the reduced temperature, $S(k)$ is the static structure factor, $\rho^{*}$ is the reduced density, $\chi_{\mathrm{T}}^{*}$ is the reduced compressibility, $c_{\mathrm{T}}^{*}$ is the reduced isothermal speed of sound, and $\tilde{c}_{\mathrm{T}}^{*}$ is the scaled reduced isothermal speed of sound. The last column gives the label of the pair of corresponding states to which the system belongs. See Table 1 .

of $\lim _{k \rightarrow 0} S(k)$. This quantity is related to the isothermal compressibility

$$
\chi_{\mathrm{T}}=\frac{1}{\rho}\left(\frac{\partial \rho}{\partial P}\right)_{T}
$$

in the following way. ${ }^{2}$

$$
\lim _{k \rightarrow 0} S(k)=\rho k_{\mathrm{B}} T \chi_{T}
$$

The isothermal compressibility is related to the isothermal speed of sound $\left(c_{T}\right)$.

$$
c_{T}=\left(\frac{1}{\chi_{T} \rho}\right)^{1 / 2}
$$

The values of these quantities for the systems studied are shown in Table 2 . The very small differences in the structure factor at small $\mathbf{k}$ for pairs of corresponding states imply larger differences in the scaled isothermal speed of sound. This will have implications for our interpretation of some of the dynamical data to be presented below.

Figure 2 shows the radial distribution functions for CS2. Similar behavior is noted for all four pairs of corresponding states investigated in this study. The differences between the structure of the two states in CS2, which are barely noticeable 


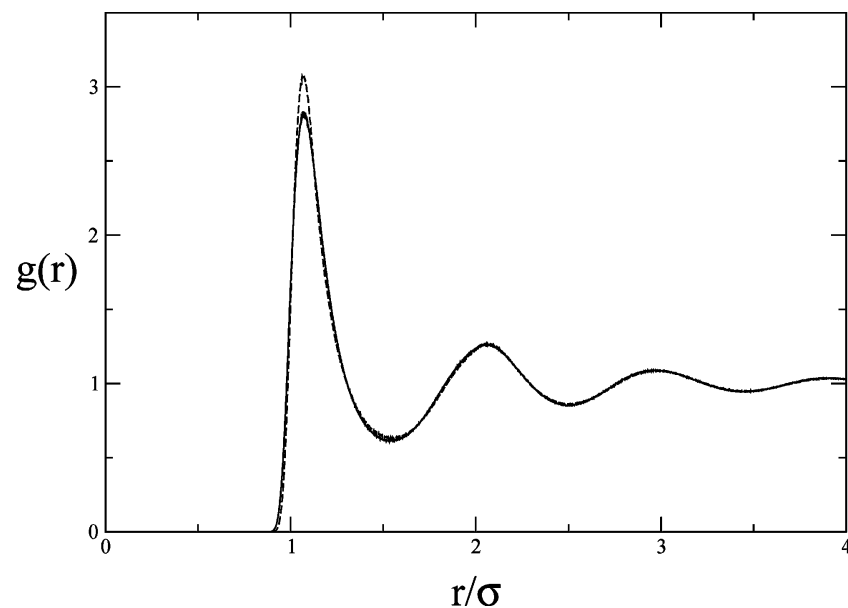

Figure 2. Comparison of the radial distribution functions for the second pair of corresponding states, CS2. RLJ system at $T^{*}=0.723$ (dashed line); LJ system at $T^{*}=1.000$ (solid line)

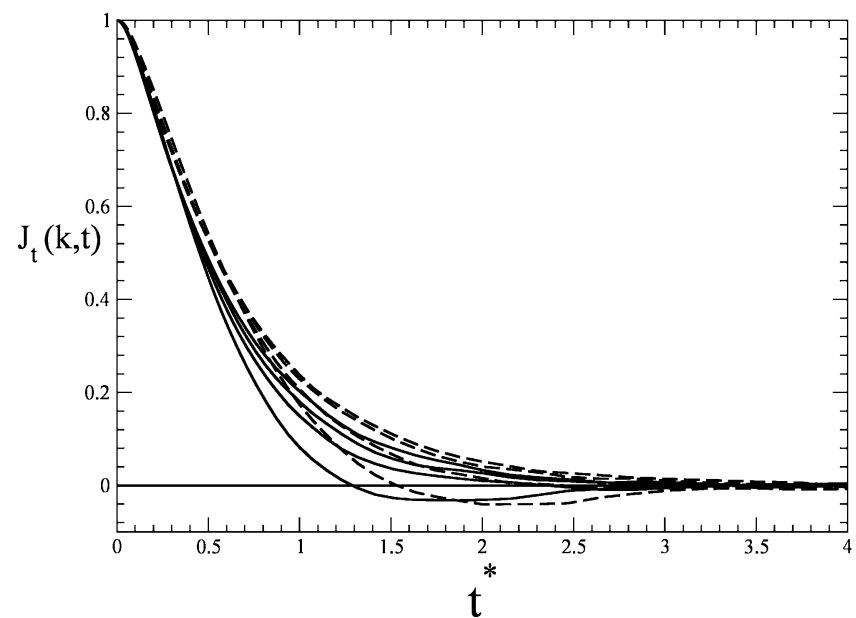

Figure 3. The normalized transverse current correlation function $J_{t}(k, t)$, for $k=0.75 \sigma^{-1}$, as a function of (unscaled) reduced time $t^{*}$ for the eight systems studied. The solid curves are data for the LJ fluids. The dashed curves are for the RLJ fluids.

in the static structure factor, are more noticeable here, primarily in the amplitude and shape of the first peak in $g(r)$.

\section{Tests of the Approximate Scaling Principle}

When data for a specific correlation function for the four pairs of corresponding states are plotted on the same graph as functions of time, the eight curves that result are typically rather different from one another. However, for some correlation functions, plotting the same data as functions of scaled time leads to four sets of two similar curves such that the similar curves in each set are for a pair of corresponding states. One of the most striking examples of this is the transverse current correlation function for small wave vectors.

Figure 3 shows the transverse current correlation function, $J_{t}(k, t)$, for $k=0.75 \sigma^{-1}$ as a function of time for the eight states studied, and Figure 4 shows the same data plotted as a function of scaled time. In the latter figure, each pair of closely spaced curves is data for a pair of corresponding states. The two highest pairs of curves are exponential at the longest times, behavior that is consistent with hydrodynamic decay of the transverse current by viscous damping. The lowest pair of curves becomes negative, consistent with the onset of shear wave propagation. This is an example of the close relationship between the

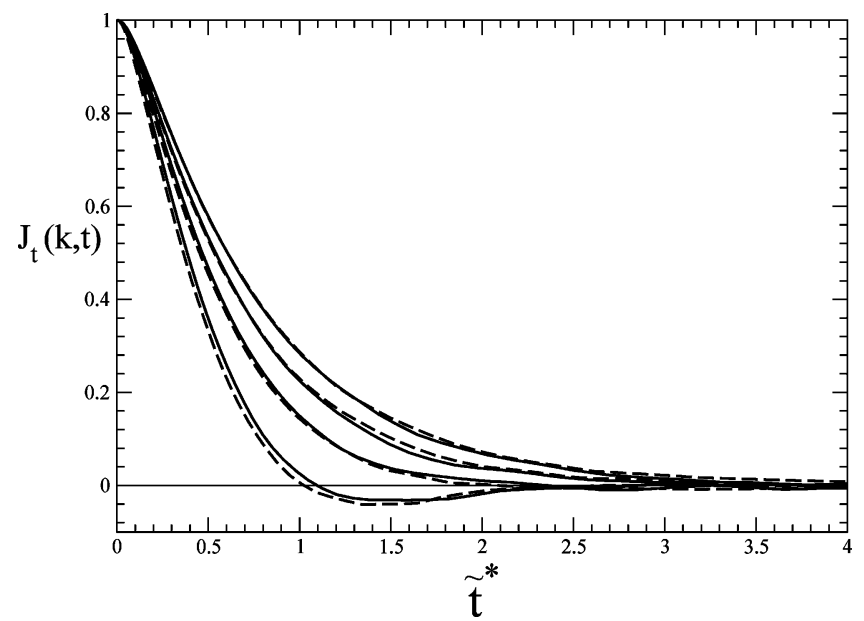

Figure 4. The normalized transverse current correlation function $J_{t}(k, t)$, for $k=0.75 \sigma^{-1}$, as a function of scaled reduced time $\tilde{t}^{*}$ for the eight systems studied. The solid curves are data for the LJ fluids at reduced temperatures of 1.554 (top curve), 1.277 (second from top), 1.000 (third from top), and 0.723 (bottom curve). The dashed curves are for the RLJ fluids at reduced temperatures of 1.277 (top), 1.000 (second from top), 0.723 (third from top), and 0.446 (bottom curve). Each pair of closely spaced curves is data for a pair of corresponding states.

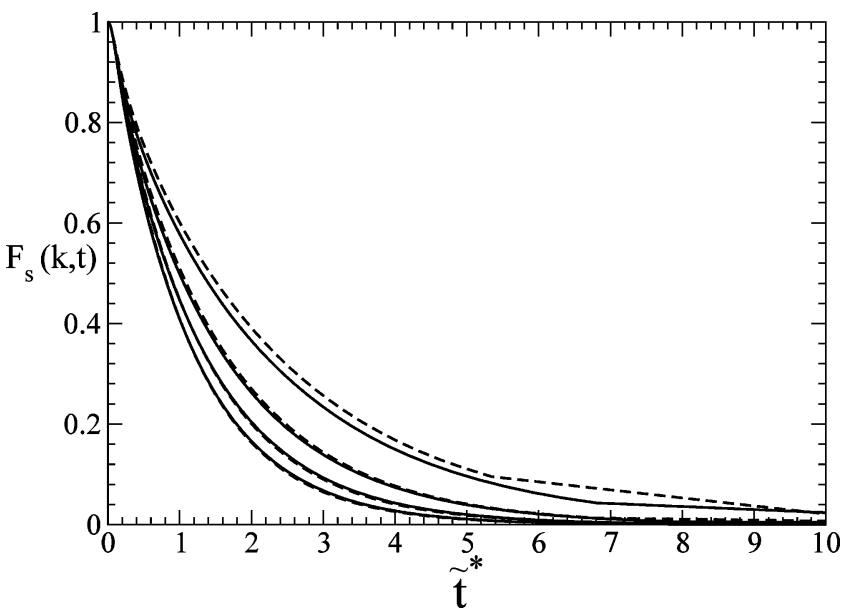

Figure 5. The incoherent intermediate scattering function $F_{\mathrm{s}}(k, t)$, for $k=3.75 \sigma^{-1}$, as a function of scaled reduced time $\tilde{t}^{*}$ for the eight systems studied. The solid curves are data for the LJ fluids at reduced temperatures of 1.554 (bottom curve), 1.277 (second from bottom), 1.000 (third from bottom), and 0.723 (top). The dashed curves are data for the RLJ fluids at reduced temperatures of 1.277 (bottom), 1.000 (second from bottom), 0.723 (third from bottom), and 0.446 (top curve). Each pair of closely spaced curves is data for a pair of corresponding states.

dynamics of corresponding states that becomes evident when the data are presented in a way suggested by the scaling principle.

In this section we compare the dynamic properties of pairs of corresponding states using this method of presenting the data.

5.1. Density Correlation Functions. 5.1.1. Incoherent Intermediate Scattering Function. The incoherent intermediate scattering functions $F_{\mathrm{s}}(k, t)$ for corresponding states are very similar for all times for all $k$ values studied. Figure 5 shows this function, for $k=3.75 \sigma^{-1}$, for all four pairs of corresponding states as a function of scaled time. Figure 2 of a previous paper ${ }^{12}$ shows this function for $k=6.75 \sigma^{-1}$. For all magnitudes of $k$ the maximum difference in the scaled incoherent intermediate scattering functions for the three higher temperature pairs of 


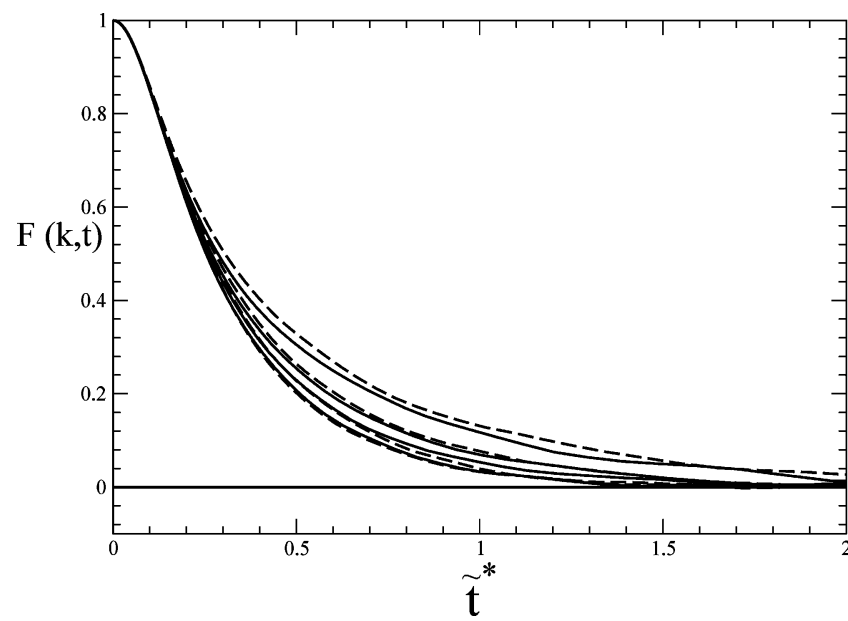

Figure 6. The normalized coherent intermediate scattering function $F(k, t)$, for $k=7.50 \sigma^{-1}$, as a function of scaled reduced time $\tilde{t}^{*}$ for the eight systems studied. See the caption of Figure 5 for more information.

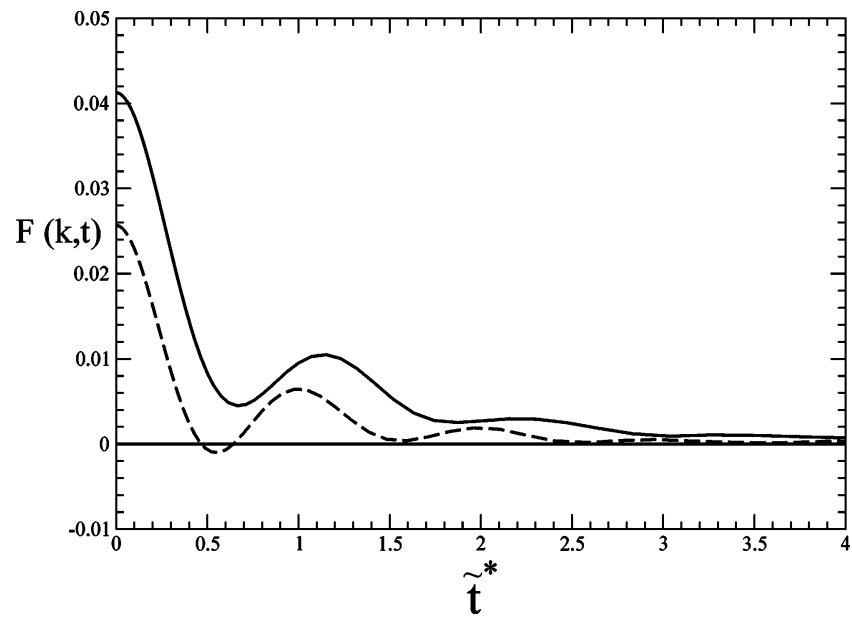

Figure 7. The coherent intermediate scattering function for $k=0.75 \sigma^{-1}$ as a function of scaled time for CS2. The data for the LJ and RLJ fluids are the solid and dashed lines, respectively.

corresponding states is approximately 0.02 and for the lowest temperature pair it is approximately 0.04 .

5.1.2. Coherent Intermediate Scattering Function. Figure 6 shows the intermediate scattering function, $F(k, t)$, for $k=$ $7.50 \sigma^{-1}$ for all four pairs of corresponding states. Figure 3 of a previous paper ${ }^{12}$ shows the same function for $k=6.75 \sigma^{-1}$. The curves for each pair of corresponding states are in close agreement for these two highest values of $k$ investigated.

As $k$ is decreased, the coherent intermediate scattering functions for corresponding states become increasingly different, and for small $k$ there is only qualitative similarity between the curves of corresponding states. Figure 7 shows the intermediate scattering functions of CS2 for $k=0.75 \sigma^{-1}$. Similar results were obtained for the other pairs of corresponding states. At low values of $k$, there are oscillations in the decay of the correlation function and the behavior of the correlators for corresponding states is clearly different.

The frequency of the oscillations at low $k$ is related to the scaled adiabatic speed of sound. This speed apparently has different values for the two states in a pair of corresponding states, which is consistent with the observation above that the scaled isothermal speeds of sound are different for corresponding states.

5.2. Current Correlation Functions and Transport Coefficients. Some of the current correlation functions of corre-

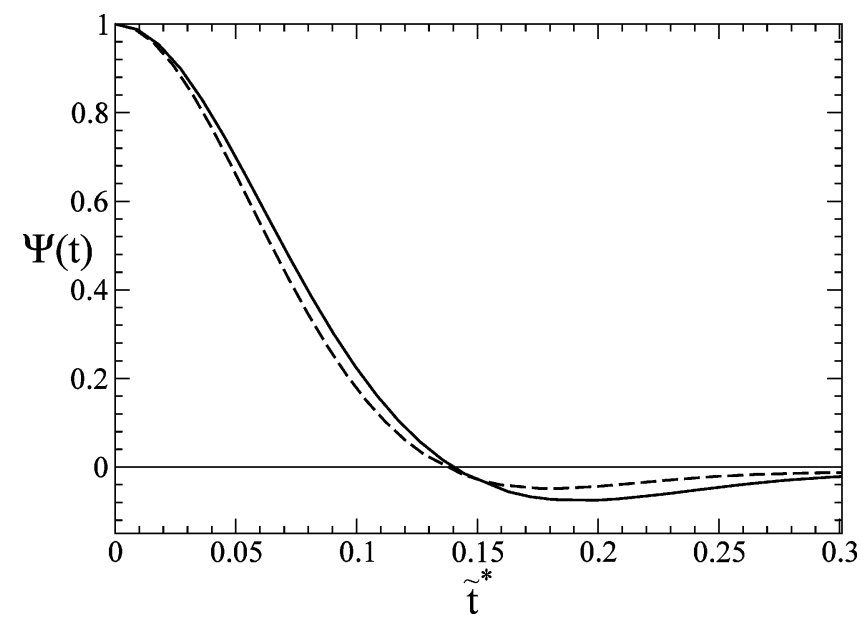

Figure 8. Velocity autocorrelation functions of CS3 for short times. The solid and dashed lines are data for the LJ and RLJ systems, respectively.

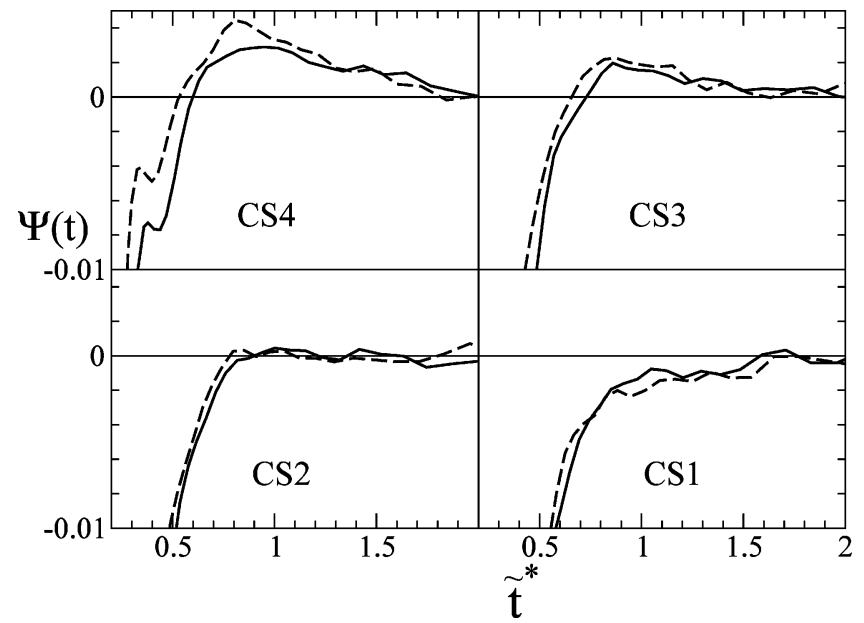

Figure 9. Velocity autocorrelation functions of the four pairs of corresponding states for intermediate and long times. The solid and dashed lines are data for the LJ and RLJ systems, respectively. For $\tilde{t}^{*}$ $\geq 1.0$, the difference between the curves for each pair of corresponding states is not statistically significant. The statistical error is approximately \pm 0.003 at all times for each of these curves.

sponding states have clear differences at short times yet have striking similarities for both intermediate and long times. For the purpose of this discussion of the current correlation functions, we shall define three different ranges of scaled reduced time $\tilde{t}^{*}$. Times in the range $0 \leq \tilde{t}^{*} \leq 0.3$ will be referred to as short times. Times such that $0.3 \leq \tilde{t}^{*} \leq 1.0$ will be referred to as intermediate times. Times such that $1.0 \leq \tilde{t}^{*}$ will be referred to as long times.

5.2.1. Velocity Autocorrelation Function. Figure 8 shows the behavior of the velocity autocorrelation function for CS3 for short times. Figure 4 of a previous paper ${ }^{12}$ shows the corresponding results for CS2. The behavior for the other corresponding states is similar. The velocity autocorrelation functions of corresponding states as functions of scaled time are clearly different for this range of times. In all the systems investigated, the initial decays of the LJ systems are slower than that for the RLJ systems and the subsequent dips below zero are more pronounced than those for the RLJ systems. Furthermore, both the times at which the minima occur and the times at which the curves first cross zero are different.

Figure 9 shows the velocity autocorrelation functions of the four pairs of corresponding states for intermediate and long 
TABLE 3: The Square of the Reduced Einstein Frequency $\left(\Omega_{0}^{*}\right)^{2}$ and of the Scaled Reduced Einstein Frequency $\left(\tilde{\Omega}_{0}^{*}\right)^{2}$ for the Systems Studied ${ }^{a}$

\begin{tabular}{ccccc}
\hline$T^{*}$ & potential & CS & $\left(\Omega_{0}^{*}\right)^{2}$ & $\left(\tilde{\Omega}_{0}^{*}\right)^{2}$ \\
\hline 0.446 & RLJ & 1 & 248 & 558 \\
0.723 & LJ & 1 & 293 & 406 \\
0.723 & RLJ & 2 & 316 & 437 \\
1.000 & LJ & 2 & 351 & 351 \\
1.000 & RLJ & 2 & 374 & 374 \\
1.277 & LJ & 3 & 402 & 314 \\
1.277 & RLJ & 4 & 422 & 331 \\
1.554 & LJ & 4 & 448 & 288
\end{tabular}

${ }^{a} T^{*}$ is the reduced temperature. The middle column gives the label of the pair of corresponding states to which the system belongs. See Table 1.

times. At intermediate times, the scaled correlators still exhibit clear differences though the qualitative trends of their temperature dependence is similar. For CS1, the functions for both the states exhibit what appears to be a long slow approach to zero from below. As the temperature is raised to CS2, this long slow approach disappears and the functions for both systems of CS2 approach zero more rapidly. For the higher temperature corresponding states (CS3 and CS4), the correlation functions rise above zero, peak, and exhibit a slow approach to zero from above. For long times $\left(\tilde{t}^{*} \geq 1.0\right)$, the scaled velocity autocorrelation functions of each pair of corresponding states are equal, i.e., the differences between them are not statistically significant.

We can understand why differences in the velocity autocorrelation functions of corresponding states must arise at short times based on an analysis of the short time expansion of the normalized velocity autocorrelation function. The result is

$$
\Psi(t)=1-\Omega_{0}{ }^{2} t^{2} / 2 !+O\left(t^{4}\right)
$$

where $\Omega_{0}$ is called the Einstein frequency. ${ }^{2}$ In terms of scaled time the expansion is

$$
\Psi(t)=1-\left(\tilde{\Omega}_{0}^{*}\right)^{2} \tilde{t}^{2} / 2 !+O\left(\tilde{t}^{4}\right)
$$

where $\left(\tilde{\Omega}_{0}^{*}\right)^{2}=\left(\Omega_{0}^{*}\right)^{2} / T^{*}$.

$\Omega_{0}{ }^{2}$ can be exactly expressed in terms of the radial distribution function $g(r)$ and the interatomic pair potential $v(r):^{2}$

$$
\Omega_{0}^{2}=\frac{4 \pi \rho}{3 m} \int_{0}^{\infty} \nabla^{2} v(r) g(r) r^{2} \mathrm{~d} r
$$

where $v(r)$ is the pair potential. Table 3 has the calculated values of $\left(\Omega_{0}^{*}\right)^{2}$ and $\left(\tilde{\Omega}_{0}^{*}\right)^{2}$ for all of the systems studied.

The values obtained for the second derivative of $\Psi(t)$ are consistent with the time dependence of the velocity autocorrelation functions at short times. The differences in the values of the scaled Einstein frequency $\left(\tilde{\Omega}_{0}^{*}\right)^{2}$ for the corresponding states illustrate that the dynamics of the scaled velocity autocorrelation functions for corresponding states have short time differences that arise from the potential as well as the static pair correlations. Even if the pair correlation function were identical for a pair of corresponding states, the $\tilde{\Omega}_{0}^{2}$ values could not in general be equal because of the large difference in $v(r) / T$ for the two systems. This is discussed more fully below.

5.2.2. Diffusion Coefficient. Despite the fact that the velocity autocorrelation functions as functions of scaled reduced time have distinct differences at short times, the integrals of the function over all times, which are related to the scaled reduced diffusion constant, are very similar within each pair of corresponding states.
TABLE 4: Scaled Reduced Diffusion Coefficients for the Eight Systems Studied ${ }^{a}$

\begin{tabular}{cccccc}
\hline$T^{*}$ & potential & CS & $\tilde{D}^{*}(\mathrm{MSD})$ & $\tilde{D}^{*}(\psi(t))$ & $\tilde{D}^{*}\left(F_{\mathrm{s}}(k, t)\right)$ \\
\hline 0.446 & RLJ & 1 & 0.033 & 0.033 & 0.033 \\
0.723 & LJ & 1 & 0.035 & 0.035 & 0.035 \\
0.723 & RLJ & 2 & 0.048 & 0.048 & 0.048 \\
1.000 & LJ & 2 & 0.049 & 0.048 & 0.050 \\
1.000 & RLJ & 3 & 0.061 & 0.061 & 0.061 \\
1.277 & LJ & 3 & 0.061 & 0.060 & 0.060 \\
1.277 & RLJ & 4 & 0.071 & 0.071 & 0.070 \\
1.554 & LJ & 4 & 0.071 & 0.070 & 0.069
\end{tabular}

${ }^{\tilde{a}} \tilde{D}^{*}$ (MSD) is calculated from the mean squared displacement as a function of time for long Times. $\tilde{D}^{*}(\psi(t))$ is calculated from the integral of the normalized velocity autocorrelation function over all times. $\tilde{D}^{*}\left(F_{\mathrm{s}}(k, t)\right)$ is calculated from the exponential decay constant for the incoherent intermediate correlation function, extrapolated to $k=0$. The third column gives the label of the pair of corresponding states to which the system belongs. See Table 1 .

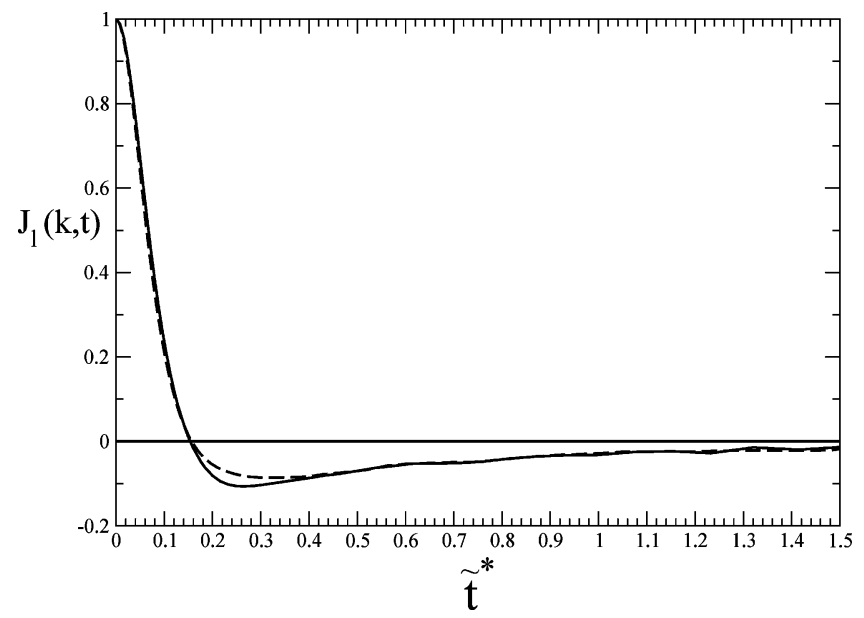

Figure 10. The normalized longitudinal current correlation function of CS2 at $k=6.75 \sigma^{-1}$. The solid and dashed lines are data for the LJ and RLJ systems, respectively.

Table 4 shows the values of the scaled reduced diffusion constant obtained from the molecular dynamics simulations in various ways. The values of the scaled diffusion coefficients of corresponding states are very close. The largest difference being approximately $7 \%$ for the lowest temperature pair of corresponding states. The other corresponding states have scaled diffusion coefficients which are within $2 \%$ of each other.

5.2.3. Self-Longitudinal Current Correlation Function. The velocity autocorrelation function is the $k=0$ value of the $k$-dependent self-longitudinal current correlation function. Comparison of this current correlation function for corresponding states gives generally the same results as noted for the velocity autocorrelation function, namely significant differences at short times, which can be related to differences in the second derivative of the correlation function at zero time, followed by often quite striking agreement at intermediate and long times.

See a later section for a discussion of integrals of the selflongitudinal current correlation function.

5.2.4. Longitudinal Current Correlation Function. Figure 10 shows the scaled longitudinal current correlation functions of CS2 for a large value of $k$. For shorter times, the correlation functions of corresponding states have significant differences similar to those of the velocity autocorrelation function. For scaled times greater than about 0.5 , the curves for corresponding states are equal to one another within the statistical error.

This pattern of behavior for short, intermediate, and long times is seen for all pairs of corresponding states but only for 


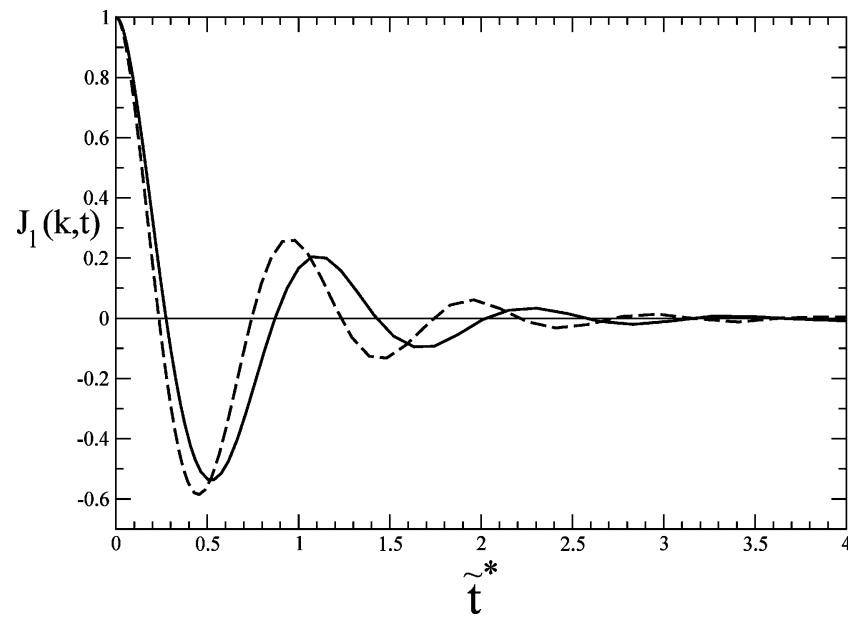

Figure 11. The normalized longitudinal current correlation function of CS2 at $k=0.75 \sigma^{-1}$ for intermediate times. The solid and dashed lines are data for the LJ and RLJ systems, respectively.

larger $k$. For smaller $k$, the functions for corresponding states are distinctly different.

Figure 11 shows the scaled longitudinal current correlation function for CS2 for the smallest value of $k$ studied. Similar behavior is seen for the other three pairs of corresponding states. For low values of $k$ (approximately $k \leq 4.50 \sigma^{-1}$ ) there are clear oscillations in the correlation functions which persist until the correlation functions are zero within the error. For these lower values of $k$ there is no evidence that scaling exists on any time scale.

The longitudinal current correlation function is simply related to the coherent intermediate scattering function.

$$
\frac{\mathrm{d}^{2} F(k, t)}{\mathrm{d} t^{2}}=-k^{2} J_{1}(k, t)
$$

The pattern of better scaling behavior for the longitudinal current correlation function at high $k$ than at low $k$ is clearly related to the corresponding pattern for the intermediate scattering function itself, as discussed above.

5.2.5. Transverse Current Correlation Function. Figure 4 shows the transverse current correlation functions for the four pairs of corresponding states for the smallest value of $k$, namely $0.75 \sigma^{-1}$. For the two higher temperatures, the correlation functions decay to zero monotonically, and the correlation functions of a pair of corresponding states are very similar. As the temperature is lowered, the correlation function dips below zero for intermediate and long times, and although the functions for corresponding states are very similar, the differences between them become larger.

Figure 12 shows the scaled transverse current correlation functions of CS2 for a larger value of $k$, namely $2.25 \sigma^{-1}$. At this value of $k$, the dips below zero are larger than at smaller $k$, consistent with the presence of highly damped shear waves.

Overall the transverse current correlation functions for corresponding states are similar but have distinct differences. These differences are similar to those noted for the longitudinal current correlation functions, namely a slightly too rapid initial decay and a slightly less pronounced negative dip for the RLJ system. Similar behavior is noted for all four pairs of corresponding states for higher values of $k$ investigated. For longer times, it appears that the curves for corresponding states become equal to one another, as observed for the longitudinal functions, but in this regime the data are very noisy, particularly for the higher values of $k$.

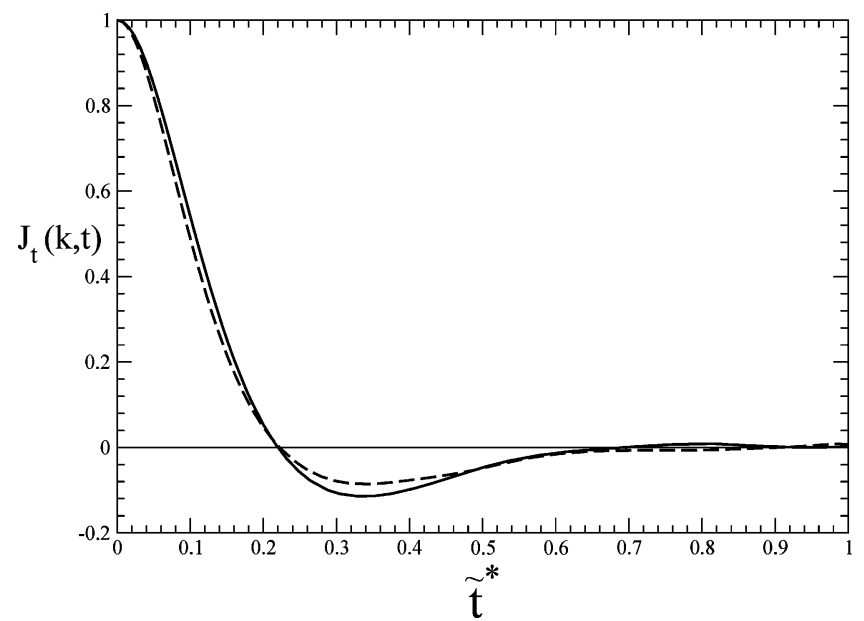

Figure 12. The normalized transverse current correlation function of CS2 at $k=2.25 \sigma^{-1}$. The solid and dashed lines are data for the $\mathrm{LJ}$ and RLJ fluids, respectively.

The change from monotonic to oscillatory decay (as either the temperature is lowered for small $k$ or the wave vector is increased at fixed temperature) has been observed previously and can be qualitatively understood on the basis of hydrodynamic theory. The linearized Navier-Stokes equations, when used to describe the decay of density fluctuations, predicts exponential, nonoscillatory decay of the transverse current correlation function. ${ }^{1}$

$$
J_{t}(k, t)=\mathrm{e}^{-\eta_{\mathrm{s}} k^{2} t / m \rho}=\mathrm{e}^{-v_{\mathrm{s}} k^{2} t}
$$

Here $\eta_{\mathrm{s}}$ is the shear viscosity and $v_{\mathrm{s}}$ is the kinematic shear viscosity. If the hydrodynamic theory is extended to include viscoelastic effects, ${ }^{1,2,25}$ the hydrodynamic theory predicts that highly damped shear waves can propagate for larger values of $k$. The propagation of the shear waves in the fluid gives rise to oscillations in the decay of the transverse current correlation function.

Thus the approximate scaling principle does not accurately describe the behavior of the transverse current correlation function for the systems we have studied in the higher $k$ regime where the dynamics can be understood as caused by the propagation of shear waves. This is analogous to what we observed above about the failure to describe the coherent intermediate scattering function at low $k$. In that case, the waves of interest were sound waves, and we had evidence of a significant difference in the scaled speed of sound within pairs of corresponding states. Presumably, a difference in the dispersion relation or damping rates for shear waves accounts for some of the deviations from the scaling principle in the transverse current correlation function. For both the coherent intermediate scattering function and the transverse current correlation function, once the damping of the waves is sufficiently strong (i.e., at high $k$ for the former and low $k$ for the latter), the corresponding states have properties that are in close agreement.

5.2.6. Integrals of the Current Correlation Functions Figure 13 shows the integral of the scaled transverse current correlation function as a function of $\tilde{t}^{*}$ for $k=4.50 \sigma^{-1}$ for all pairs of corresponding states. More precisely, the quantity plotted is

$$
\int_{0}^{\tilde{t}^{*}} \mathrm{~d} \tilde{t}^{*} \tilde{J}_{t}^{*}\left(\mathbf{k}, \tilde{t}^{* \prime}\right)
$$

where the integrand is the scaled transverse current correlation function as a function of scaled time. The integration is performed with regard to scaled time, and the upper limit of 


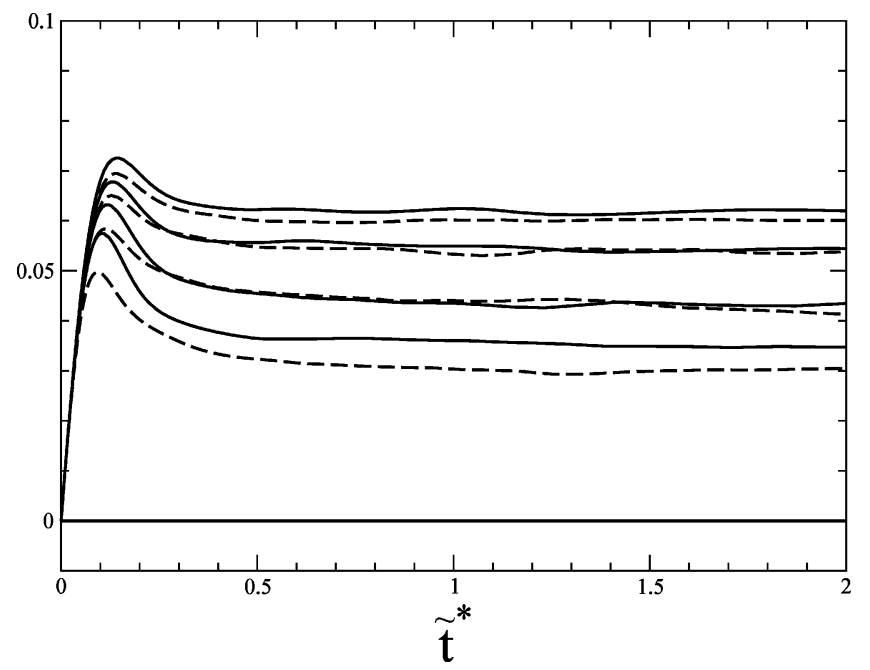

Figure 13. The integral of the scaled transverse current correlation function for the eight systems studied at $k=4.50 \sigma^{-1}$. (See the text for the precise meaning of the area.) The solid curves are data for the $\mathrm{LJ}$ fluids. The dashed curves are data for the RLJ fluids. Each pair of closely spaced curves is data for a pair of corresponding states, with CS1-CS4 appearing in order from bottom to top.

the integral is the abscissa of the graph. The integrals hit a plateau value beyond which the data for the correlators is zero within the noise. For each pair of corresponding states, the values of the plateaus are very similar. Similar results are also seen for smaller $k$. These similarities are not surprising for the two lowest $k$ values at the highest temperatures, since the correlators themselves are very close. However, for the $k$ value in Figure 13, the correlators for corresponding states have clear differences yet the integrals of the correlators are very nearly equal. For higher $k$, the data are too noisy to tell whether this trend continues.

The integral of the velocity autocorrelation function shows similar behavior. In this case the area is related to the scaled reduced self-diffusion coefficient. See Table 4.

The integral of the self-longitudinal current correlation function shows a closely related behavior. In this case the long time limiting value of the integral is identically zero for any system, so the equality of the long time limiting values is a trivial result. However, the integrals for corresponding states become equal and stay equal (within the statistical error) before the integrals themselves become zero.

5.2.7. Shear Viscosity. For the three highest temperature pairs (CS2, CS3, and CS4) of corresponding states, the transverse current correlation function appears to decay exponentially at the lowest value of $k$ investigated. Using eq 5, we can extract values for the shear viscosity coefficient from the data. Table 5 shows the resulting values of the scaled kinematic viscosity $\tilde{v} *_{\mathrm{s}}$ and the scaled shear viscosity coefficient $\eta *_{\mathrm{s}}$. (Since we can do this for only one small value of $k$, there is no way to extrapolate the results to $k=0$. The results quoted in the table are based on the assumption that the macroscopic kinematic shear viscosity for long wavelength hydrodynamic behavior can be extracted from the decay of the transverse current correlation function for a wavevector of $k=0.75 \sigma^{-1}$, which corresponds to a wavelength of $8.38 \sigma$.)

For the three pairs of corresponding states for which we can obtain the shear viscosity in this way, the two states in a pair have shear viscosity coefficients that are equal to within $3 \%$ or less.

The Stokes-Einstein relationship for the diffusion coefficient and shear viscosity coefficient of a hard sphere fluid, when
TABLE 5: The Scaled Reduced Kinematic Viscosity Coefficient $v_{\mathrm{s}}$ and Scaled Reduced Shear Viscosity Coefficient $\tilde{\eta}_{\mathrm{s}}$ for Six of the Corresponding States Studied, Extracted from the Time Dependence of the Transverse Current Autocorrelation Functions Obtained from Computer Simulations ${ }^{a}$

\begin{tabular}{lccccc}
\hline \multicolumn{1}{c}{$T^{*}$} & potential & CS & $\tilde{v}_{\mathrm{s}}^{*}$ & $\tilde{\eta}_{\mathrm{s}}^{*}$ & $1 / 2 \pi \tilde{\eta}^{*} \tilde{D}^{*}$ \\
\hline 0.723 & RLJ & 2 & 3.90 & 3.29 & 1.01 \\
1.0 & LJ & 2 & 3.86 & 3.25 & 1.02 \\
1.0 & RLJ & 3 & 2.90 & 2.45 & 1.06 \\
1.277 & LJ & 3 & 2.98 & 2.52 & 1.05 \\
1.277 & RLJ & 4 & 2.45 & 2.07 & 1.08 \\
1.554 & LJ & 4 & 2.48 & 2.09 & 1.09
\end{tabular}

${ }^{a}$ See the discussion in the text for the assumptions behind the analysis. $\tilde{D}^{*}$ is the scaled reduced diffusion coefficient from Table 4. The third column gives the label of the pair of corresponding states to which the system belongs. See Table 1 . See the text for the interpretation of the last column of numbers.

expressed in terms scaled quantities, is $\tilde{\eta}^{*}=1 / 2 \pi d^{*} \tilde{D}^{*}$, where $d^{*}$ is the effective hard sphere diameter in units of $\sigma$. This formula is derived by using slip boundary conditions. In Table 5 we give values of $1 / 2 \pi \tilde{\eta}^{*} \tilde{D}^{*}$. If the Stokes-Einstein relationship were correct for these systems, this quantity should equal $d^{*}$. The values in the table are quite reasonable, with all of them being slightly larger than 1 but less than $2^{1 / 6}$, which is the maximum distance of the repulsive forces in the LJ and RLJ potentials.

\section{Summary and Discussion}

We have examined four pairs of corresponding states to investigate the similarities and differences of the scaled dynamical properties within each pair. The states in a pair have the same density, different temperatures, and very different interatomic potentials, but very similar equilibrium pair correlation functions. The approximate scaling principle conjectures that the scaled dynamics of the two states in a pair should be very similar.

We have seen significant similarities for many but not all dynamical properties. The similarities are greatest for the incoherent intermediate scattering function, the velocity autocorrelation function and self-longitudinal correlation function for intermediate and long times, the coherent intermediate scattering function for large wave vectors, the longitudinal current correlation function for large wave vectors and intermediate and long times, and the transverse current correlation function for small wave vectors and higher temperatures. The differences are greatest for (1) both the coherent intermediate scattering function and the longitudinal current for small wavevectors, (2) all the current correlation functions at short times, and (3) the transverse current correlation function for larger wave vectors and/or low temperatures. Despite the fact that the current correlation functions of corresponding states have different behavior at short times (with the exception of the transverse current for small wavevector), the integrals of these functions with regard to scaled time from $\tilde{t}^{*}=0$ to a long time are very similar for corresponding states. Moreover, the scaled diffusion coefficients and scaled shear viscosity coefficients of corresponding states are very similar.

To understand this pattern of results, one might ask the question of why the scaling principle works when it does. However, the following alternative approach appears to be worthwhile. The similarities in the dynamics of a pair of corresponding states are so striking for so many properties that it is reasonable to accept the approximate scaling principle as 
the "default" behavior that is to be expected and then to ask why the principle fails when it does.

Failure of the approximate scaling principle for a specific dynamical property is presumably due to one or both of the following: (1) The specific dynamical property is very sensitive to the small differences in the static pair correlation function. (2) The specific dynamical property is sensitive to multiparticle correlation functions that may differ significantly in the two states of a pair.

For each of the three situations outlined above in which the approximate scaling principle was most incorrect, it is possible to understand the basis for the failure.

(1) The approximate scaling principle does not work well for the coherent intermediate scattering function and the closely related longitudinal current correlation function for small wave vectors. The dynamics of these functions is qualitatively understood on the basis of linearized hydrodynamic theory. The detailed time dependent behavior is influenced by the dispersion relation for sound waves and by the speed of sound. The speed of sound of a material is directly related to its compressibility. The compressibility is related to the $k \rightarrow 0$ limit of the static $S(k)$. The numerical values of the compressibility of the two states in a pair are very different, even though they have very similar structure factors at small $k$. Thus, the propagation of sound waves represents a property of a fluid that is very sensitive to this aspect of the structure. (Note that this sensitivity argument does not apply to larger wave vectors because the linearized hydrodynamic theory is not valid for large $k$, and indeed for larger wave vectors the approximate scaling principle holds well for these two correlation functions.)

(2) The approximate scaling principle does not work well for the current correlation functions at short times. The current correlation functions for short times can be expressed in terms of static averages that involve the potential as well as the static pair correlation function. For the velocity autocorrelation function, the expressions are eqs 3 and 4, and similar but more complicated expressions can be derived for the other current correlation functions. Since these expressions involve the interatomic potential directly, the similarity of the static pair correlation functions for a pair of corresponding states is not sufficient to guarantee similar values for the initial second derivatives. In the context of fully renormalized kinetic theory, this means that the initial values of the current correlation functions must be sensitive to other many body static correlation functions, not just the pair correlation function, and those many body static functions must differ within a pair of corresponding states if the pair has very different interatomic potentials. (However, for longer times, the same current correlation functions are apparently much less sensitive to many body static correlation functions.)

(3) The approximate scaling principle does not work well for the transverse current correlation function for large wave vectors. For large wave vectors, a generalized hydrodynamic theory predicts the possibility of highly damped transverse waves. Their dynamics must be sensitive to the dispersion relationship for transverse waves. It is reasonable to suppose that these dispersion relationships might differ significantly in a pair of corresponding states, despite the fact that the static pair correlation function is very similar in the two states of the pair, just as is the case for sound waves. (Note that this sensitivity argument does not apply for smaller wave vectors, which cannot support transverse waves, and indeed for small wave vectors the corresponding states principle holds well for the transverse current.)
The approximate scaling principle holds for a variety of dynamical properties. For properties for which it is inaccurate, we can usually understand the reason. The results show that some, but not all, dynamical properties of a fluid are very sensitive to the changes in the interatomic potential that keep the pair correlation function approximately unchanged, whereas others are much less sensitive. It is interesting that transport coefficients and integrals of the current correlation functions appear to be insensitive to changes in the potential that leave the pair correlation function approximately unchanged. This suggests that it will be easier to develop a kinetic theory for transport coefficients than for the current correlation functions, and it may help to explain why hard sphere models have been so useful in explaining transport properties of liquids that clearly have potentials that differ significantly from the hard sphere potential.

Acknowledgment. We thank Madhav Ranganathan for numerous helpful discussions. This work was supported by the National Science Foundation through grants CHE-9734893, CHE-0010117, and CHE-0408786.

\section{References and Notes}

(1) Boon, J. P.; Yip, S. Molecular Hydrodynamics; McGraw-Hill: New York, 1980.

(2) Hansen, J.-P.; McDonald, I. R. Theory of Simple Liquids, 2nd ed.; Academic: London, UK, 1986.

(3) Allen, M. P.; Tildesley, D. J. Computer Simulation of Liquids; Oxford: New York, 1989.

(4) Balucani, U.; Zoppi, M. Dynamics of the Liquid State, 1st ed.; Oxford: New York, 1994.

(5) Gross, E. P. Ann. Phys. (N.Y.) 1972, 69, 42.

(6) Mazenko, G. F. Phys. Rev. 1974, A9, 360.

(7) Boley, C. D. Phys. Rev. 1975, A11, 328.

(8) Mazenko, G. F.; Yip, S. In Statistical Mechanics. Part B: TimeDependent Processes; Berne, B. J., Ed.; Plenum: New York, 1977.

(9) Andersen, H. C. J. Phys. Chem. B 2002, 106, 8326.

(10) Andersen, H. C. J. Phys. Chem. B 2003, 107, 10226.

(11) Andersen, H. C. J. Phys. Chem. B 2003, 107, 10234.

(12) Young, T.; Andersen, H. C. J. Chem. Phys. 2003, 118, 3447.

(13) Levesque, D.; Verlet, L. Phys. Rev. 1970, 2, 2514.

(14) Levesque, D.; Verlet, L.; Kürkijarvi, J. Phys. Rev. 1973, A7, 1690.

(15) Protopapas, P.; Andersen, H. C.; Parlee, N. A. D. J. Chem. Phys. 1973, 59, 15 .

(16) Protopapas, P.; Andersen, H. C.; Parlee, N. A. D. Chem. Phys. 1975, 8,17 .

(17) Ashurst, W. T.; Hoover, W. G. Phys. Rev. 1975, A11, 658.

(18) Rosenfeld, Y. Phys. Rev. 1977, A15, 2545.

(19) Rosenfeld, Y. Chem. Phys. Lett. 1977, 48, 467.

(20) Dzugutov, M. Nature 1996, 381, 137.

(21) Hoyt, J. J.; Asta, M.; Sadigh, B. Phys. Rev. Lett. 2000, 85, 594.

(22) Our use of the phrase "corresponding states" should be distinguished from previous uses of the term; see, e.g.: Pitzer, K. S. J. Chem. Phys. 1939, 7, 583. Hirschfelder, J. O.; Curtiss, C. F.; Bird, R. B. Molecular Theory of Gases and Liquids; Wiley: New York, 1954. The "principle of corresponding states" relates the static and/or dynamic properties of two (or more) fluids based on a similarity of their intermolecular potentials. It shows how to identify, for any thermodynamic state of one fluid, the corresponding state of the other fluid whose properties are related to those of the first fluid with a proper scaling of the variables. In our case, a pair of corresponding states is one fluid in one thermodynamic state and another fluid in another thermodynamic state such that the two states have the same density and very similar pair correlation functions, when the length is scaled appropriately. Our approximate scaling principle then postulates a scaling relationship among the dynamic (but not the static) properties of the pair.

(23) Weeks, J. D.; Chandler, D.; Andersen, H. C. J. Chem. Phys. 1971, $54,5237$.

(24) Swope, W. C.; Andersen, H C.; Behrens, P. H.; Wilson, K. R. J. Chem. Phys. 1982, 76, 637.

(25) Berne, B. J.; Pecora, R. Dynamic Light Scattering; Dover: New York, 2000 